\title{
Exudates Detection with DBSCAN Clustering and Back Propagation Neural Network
}

\author{
Shantala Giraddi \\ Computer Science Department \\ B.V.B College of Engineering and \\ Technology \\ Hubli, India
}

\author{
Jagadeesh Pujari, Ph.D \\ Information Science Department \\ S.D.M. College of Engineering and \\ Technology \\ Dharwad, India
}

\author{
Shraddha Giraddi \\ Computer Science Department \\ B.V.B College of Engineering and \\ Technology \\ Hubli, India
}

\begin{abstract}
Diabetic Retinopathy (DR) is the third biggest cause of blindness in India. Hard exudates are the primary signs of DR. In this paper the authors propose a novel hybrid mechanism for the detection of Exudates based DBSCAN clustering algorithm. Unlike other clustering algorithms, DBSCAN clustering does not require the number of clusters to be specified. Classification of regions is being done using a system based on Back propagation Neural Network. The authors assessed the performance of algorithm using one of the publicly available databases DIARETDB1. Sensitivity of $90 \%$ and a specificity of $85 \%$ are achieved using a lesion based performance evaluation criterion and an accuracy of $100 \%$ is obtained on image based performance evaluation criterion.
\end{abstract}

\section{Keywords}

Hard exudates, DBSCAN clustering, Diabetic Retinopathy, Back propagation neural network

\section{INTRODUCTION}

Diabetic Retinopathy (DR) is a complication of Diabetes. Different kinds of abnormal lesions are caused by DR. Alterations in blood vessel diameter, micro aneurysms, hard exudates, cotton wool spots, haemorrhages and Neovascularisation are key symptoms of Diabetic Retinopathy. Among these, Exudates are the primary signs of DR.

In ophthalmology, eye fundus examination is highly preferred for diagnosing the abnormalities and the follow-up of the development of the eye disease. Periodic screening and automated early detection can prevent the blindness. The screening program produces an enormous amount of retinal images since diabetic patients typically have both their eyes examined at least once in a year. The manual screening of these images requires both high financial cost and human resource. Nowadays, several approaches have been considered to build automatic computer-based screening programme. Automated detection mechanism can reduce the workload and increase the follow-up management of diabetic patients.

Digital color fundus images are widely used by ophthalmologists for diagnosing Diabetic Retinopathy. Figure 1 shows fundus image with various abnormalities of Diabetic Retinopathy

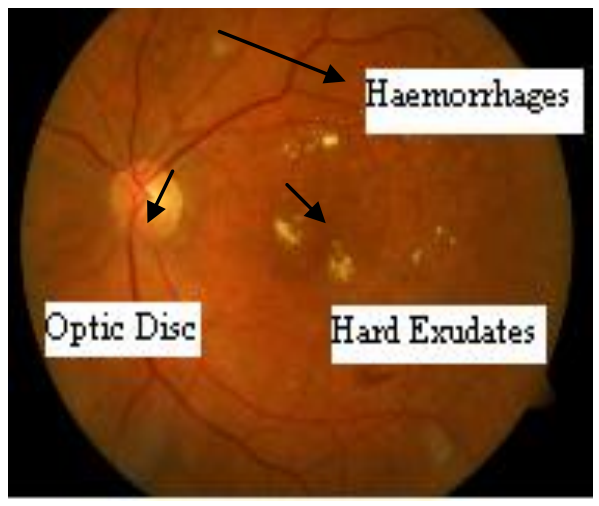

Figure 1. Diabetic retinopathy image with various typical components

The growing incidence of diabetes and lack of specialists increase the workload of physicians. High cost of treatment prevents many patients from receiving effective treatment. Automatic detection of clinical signs of DR can help ophthalmologists in the diagnosis of the disease and with the subsequent cost and time savings.

\section{LITERATURE SURVEY}

Sinthunayothin et.al [1] used recursive region growing which yielded hard exudates as well as optic disc. Optic disc was removed by identifying the area with highest variation in intensity of adjacent pixels. Akara and Uyyanonvara [2] used Fuzzy C-means and morphological based segmentation for diagnosing the exudates from low-contrast images. Coarse segmentation is done using FCM and finer segmentation is done using morphological reconstruction. Akara[3] performed a series of experiments and did a comparative study between mathematical morphology, fuzzy c-means clustering, Naive Bayesian classifier, Support Vector Machine, Nearest Neighbour Classifier and detected exudates were validated with ophthalmologists hand drawn ground-truths. Highest sensitivity $97.29 \%$ is obtained with FCM. Hussain F.Jaafar et.al [4] proposed a method based on top-down image segmentation and local thresholding by a combination of edge detection and region growing Grading of hard exudates is performed. Maria Garcia.et.al [5] used a combination of global and local thresholding for segmentation of candidate exudates regions. Candidate regions are used for the training of RBF networks. The trained network performed the pixel wise classification. R.Vijayamadheswaran [6].et.al used contextual clustering (CC) for feature extraction and extracted features were used as input to RBF network. Neera Singh and Ramesh Chadra [7] used fuzzy $\mathrm{C}$-means clustering. Features like colour, size, edge and texture are extracted from the resulting clusters. A system based Back propagation neural network is used to classify pixels into exudates and non-exudates. Nathan Silberman et.al [8] extracted SIFT features from a limited ROI region which is a 
horizontal conic area spanning from the location of optic disc outward toward centre of the retina. These features are used to train the Gaussian Support Vector Machine to label individual patches of image. Vijayakumari and SuriyaNaraynan [9] used template matching for optic disc detection. Authors used sobel edge detector to find objects with sharp edges and used enhanced MDD classifier to find yellowish objects. Asha gowda et.al [10] attempted a method in which Back propagation Neural Networks are used for exudates detection. Features like Hue, Intensity, Standard deviation of intensity, distance between mean of optic disc pixels and pixels of exudates and non-exudates and mean intensity have been used as inputs to train the neural network .Ivo soares et.al [11] used combination of morphological operators and adaptive thresholding. The authors claim that the method is unaffected by contrast changes, non-uniform illumination and variable background resulting in correct detection of exudates.

Among the methods proposed so far, region growing and thresholding methods have the difficulty of determining the threshold value. Machine learning approaches which perform pixel wise classification are computationally expensive. Edge based approaches have the difficulty of differentiating the pixels belonging to vessels and pixels belong to edges of exudates. In Clustering based approaches, it is difficult to specify the number of clusters.

In this paper we propose a novel method based on DBSCAN clustering in which number of clusters need not be specified. The two parameters are eps - the distance between the pixels and Minpts is the minimum number of points. A pixel is considered to be core pixel if it has Minpts of pixels within eps distance. The result of DBSCAN may yield false positives, which need to be classified further. For the classification of these regions we have used a system based on Back propagation Neural Network.

\section{MATERIALS AND METHODS}

We have used digital images from publicly available databases DIARETDB0 and DIARETDB1. In addition to these images we have also used images from Vasan Eye care centre, Hubli for our study.

The methodology consists of the following steps

\section{Image Acquisition}

2. Image Pre-processing

3. Optic disc removal

3. Segmentation using DBSCAN clustering

4. Feature Extraction

\section{Classification by Back propagation Neural}

\section{Network}

\subsection{Image Acquisition}

In this study we have considered two well known databases DIARETDB0 and DIARETDB1. DIARETDB0 is having 130 images and DIARETDB1 is having 89 images. The images are of the size 1500X1152.The images are captured with $50 \mathrm{deg}$ field of view.

\subsection{Image Pre-processing}

The images obtained from fundus camera are of low contrast and need some contrast enhancement. Each image is contrast enhanced by CLAHE. Since CLAHE increases even the noise, image is median filtered using a filter of size $3 \times 3$.

\subsection{Optic Disc Removal}

Since optic disc has the same characteristics as the exudates, it needs to be detected and eliminated from further analysis. The authors have used combination of recursive region growing and Active Contour method for optic disc localization and boundary detection. The procedure is given below:

a. Initialize seed points

b. Perform region growing algorithm

c. Perform connected component analysis

d. Find the centroid of component

e. Initialize an ACM curve by a circle of 50 Pixels

f. Allow the curve to evolve

g. Map the curve to original image

The results of image pre-processing and optic disc removal are shown in the figure 2 and figure 3.
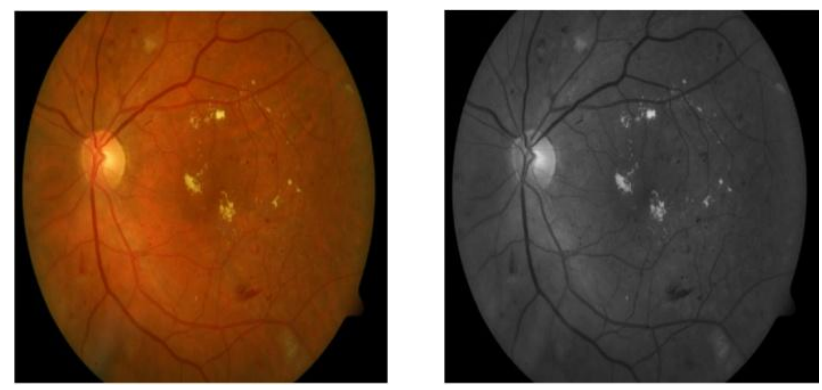

a.Original RGB image

b.Green channel of image
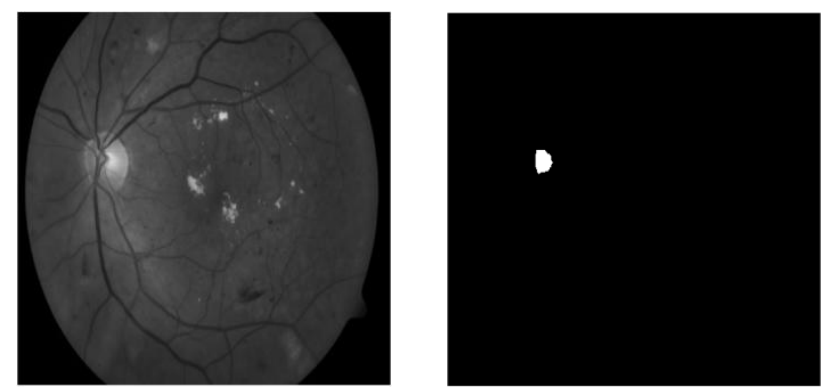

c.Contrast enhanced by

d.Result of region growing CLAHE

Figure 2. Result of Preprocessing and Region growing

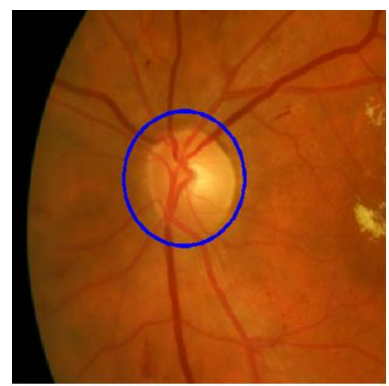

a. Initial curve placed

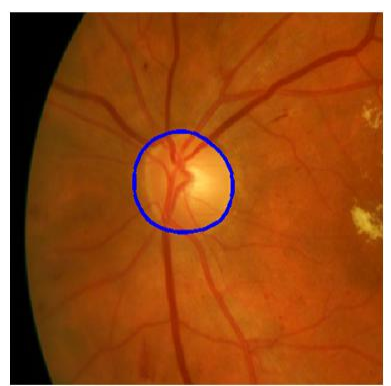

b. Curve after 200 iterations
Figure 3. Result of optic disc detection by Active Contour method 


\subsection{Segmentation using DBSCAN Clustering}

In this study we apply DBSCAN clustering to image segmentation problem. DBSCAN is a density based clustering algorithm that is designed to discover areas of high density that are separated from each other by area of low density [12]. It can identify clusters in large spatial data set. It has two parameters eps - the distance between two points and MinPts - minimum number of points to form a cluster. It starts with an arbitrary starting point that has not been visited. This point's epsilon neighborhood is retrieved. If it has sufficiently many points, a cluster is started. Otherwise this point is labeled as noise. This point might later be found in a sufficiently sized epsilon environment of a different and hence is made part of a cluster.

We have chosen $\mathrm{L}^{*} \mathrm{a} * \mathrm{~b}$ color space for clustering. Three features are selected as input to DBSCAN clustering algorithm. The features used are,

a. The Pixels chromaticity value *a after preprocessing

b. The Pixels chromaticity value *b after preprocessing.

c. The standard deviation of preprocessed *a value in a window around the pixel.

The window size is chosen $3 \times 3$. We are using standard deviation since the exudates are more textured than nonexudates.

Fine tuning of the algorithm parameters is being done using 15 representative images. Based on the performance of clustering algorithm on these images Eps is set to 1.3 and MinPts is set to 50 .

DBSCAN is designed to discover noise as well as clusters in the spatial data set. The number of clusters obtained from the algorithm is much more than number of color regions that can be identified by humans as shown in. Fig.4. Along with exudates, there are some bright regions that have been segmented as exudates. Therefore some post processing is necessary to classify these false positives as non-Exudates.

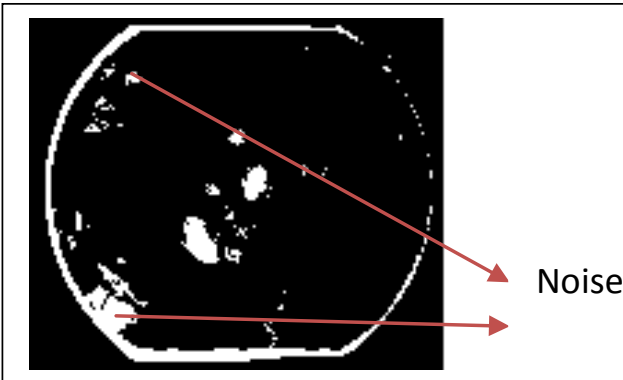

Figure 4. Result of DBSCAN clustering having false positives

In order to further classify theses candidate regions, we have extracted various first order features from these regions. The features extracted are shown in Table 1. Due to the high dimensionality of the feature vectors, dimensionality reduction has been performed with use of various feature selection algorithms. Feature selection methods select a subset of the original set of features. They involve use of search algorithms and the maximization of a given evaluation function, which corresponds to the separability between the class populations. In this work, the evaluation function $\mathrm{J} 1$ used by the algorithms is a distance criterion, based on the scatter matrices: $\operatorname{trace}\{1\} J 1=S w-S b$

Where $\mathrm{Sw}$ and $\mathrm{Sb}$ are respectively the within-class and between-class scatter matrices, calculated from the covariance matrices of the class populations. We used Sequential Floating Forward Search (SFFS) for optimal feature selection. The subset with seven features Area, Eccentricity, Solidity, Extent, Max green, Max Intensity, and Max red has a minimum classification error.

\subsection{Classification using Back propagation neural network}

The candidate regions resulting from clustering algorithm are further classified by a system based on Backpropogation Neural Network. The back propagation algorithm is a generalization of the least mean square algorithm that modifies network weights to minimize the mean squared error between the desired and actual outputs of the network. The back propagation algorithm trains a given feed forward multilayer neural network for a given set of input Patterns with known classifications. When each entry of the sample set is presented to the network, the network examines its output response to the sample input pattern. The output response is then compared to the Known and desired output and the error value are calculated. Based on the error, the connection weights are adjusted.

Table 1. Features extracted from lesions for further Classification

\begin{tabular}{|l|l|l|l|}
\hline 1 & Area & 12 & Mean(*a) \\
\hline 2 & orientation & 13 & Variance(*a) \\
\hline 3 & Eccentricity & 14 & Energy(*a) \\
\hline 4 & Solidity & 15 & Mean(*b) \\
\hline 5 & Extent & 16 & Variance(*b) \\
\hline 6 & Mean(green) & 17 & Energy(*b) \\
\hline 7 & Variance(green) & 18 & $\operatorname{Max}($ green $)$ \\
\hline 8 & Energy(green) & 19 & $\operatorname{Max}($ Intensity) \\
\hline 9 & Mean(Intensity) & 20 & $\operatorname{Max}(* a)$ \\
\hline 10 & Variance(Intensity) & 21 & $\operatorname{Max}(* b)$ \\
\hline 11 & Energy(Intensity) & & \\
\hline
\end{tabular}

\subsubsection{Neural Network architecture}

Deciding the number of neurons in the hidden layers is very important part of deciding your overall neural network architecture. Though these layers do not directly interact with the external environment, they have a tremendous influence on the final output. Both the number of hidden layers and the number of neurons in each of these hidden layers must be carefully considered. Using too few neurons in the hidden layers will result in something called under fitting. Under fitting occurs when there are too few neurons in the hidden layers to adequately detect the signals in a complicated data set. Using too many neurons in the hidden layers can result in several problems. First, too many neurons in the hidden layers may result in over fitting. Over fitting occurs when the neural network has so much information processing capacity that the limited amount of information contained in the training set is not enough to train all of the neurons in the hidden layers. A second problem can occur even when the training data is sufficient. An inordinately large number of neurons in the hidden layers can increase the time it takes to train the network. The amount of training time can increase to the point that it is 
impossible to adequately train the neural network. Obviously, some compromise must be reached between too many and too few neurons in the hidden layers.

\subsubsection{Performance analysis based on hidden nodes:} In order to choose right number of hidden nodes and hidden layers, authors have done performance analysis of classifiers with different number of hidden nodes and layers. Table 2 shows results of each classifier. As seen in Table 2, BPNN with 10 nodes in the hidden layer gives the minimum error. For training the network we have chosen the following parameters:

Training function-sigmoid, MSE $=0.01$, Learning rate $=0.4$. As we increase the number of hidden nodes, we get better performance. As the neural network architecture expands, training time increases. If accuracy is more important we should use more hidden nodes, on the other hand if time is a critical factor, we should use less hidden nodes and hidden layers. Figure 5 shows comparative performance analysis of BPNN with different number of hidden nodes. Highest classification accuracy is obtained with architecture [7-5-1].

Table 2. Performance analysis of Back propagation neural network with different number of hidden neurons

\begin{tabular}{|c|c|c|c|}
\hline $\begin{array}{c}\text { \#Hidden } \\
\text { Layer }\end{array}$ & Architecture & CRR \% & MSE \\
\hline 1 & {$[7-1-1]$} & 80 & 0.4553 \\
\hline 1 & {$[7-2-1]$} & 70 & 0.3749 \\
\hline 1 & {$[7-3-1]$} & 75 & 0.3687 \\
\hline 1 & {$[7-4-1]$} & 75 & 0.4346 \\
\hline 1 & {$[7-5-1]$} & 89 & 0.3224 \\
\hline 1 & {$[7-6-1]$} & 83 & 0.14847 \\
\hline 1 & {$[7-7-1]$} & 83 & 0.51852 \\
\hline 1 & {$[7-8-1]$} & 85 & 0.24955 \\
\hline 2 & {$[7-4-3-1]$} & 88 & 0.287270 \\
\hline 2 & {$[7-4-4-1]$} & 87 & 0.202368 \\
\hline
\end{tabular}

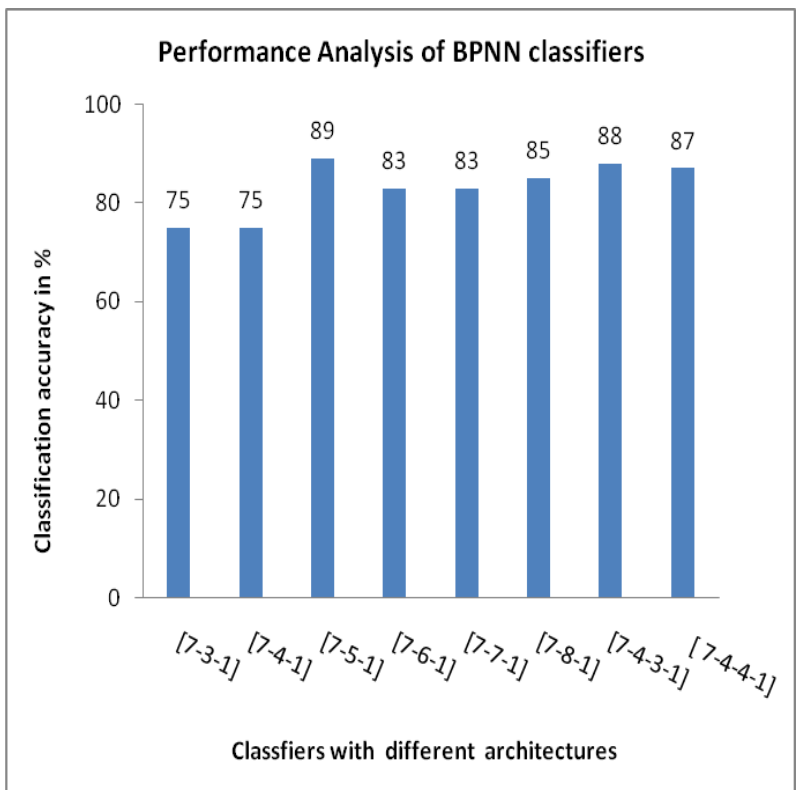

Figure 5. Performance analysis of BPNN with different number of hidden nodes
Table 3. Results of Clustering and classification based on Image based criterion

\begin{tabular}{|l|c|c|c|}
\hline Data base & $\begin{array}{l}\text { No of } \\
\text { images }\end{array}$ & sensitivity & specificity \\
\hline DIARETB1 & 20 & $100 \%$ & $92 \%$ \\
\hline
\end{tabular}

\section{CONCLUSION}

Due to lack of reliable way to measure the performance, it is difficult to compare our results to those reported in the literature. In this study, a database with manually annotated HE by experts has been used. DBSCAN algorithm combined with Back Propagation Neural Network provides effective mechanism for early detection of hard exudates. DBSCAN algorithm does not need the number of clusters to be specified.

We have two criteria for algorithm evaluation. Lesion based accuracy and image based accuracy. We are able to detect exudates in all the 20 images. Sensitivity of $100 \%$ and specificity of $92 \%$ were obtained in terms of image based accuracy. In terms of lesion based accuracy sensitivity of $90 \%$, specificity of $85 \%$ was obtained. Some false positives were found in some images. Further improvements can be done on the proposed algorithms when more suitable data is available.

\section{REFERENCES}

[1] C. Sinthanayothin, "Automated detection of diabetic retinopathy on digital fundus images", Diabetic Medicine, 19, pp 105-112, 2002.

[2] Akara Sopharak, Bunyarit Uyyanonvara, "Automatic exudates detection from diabetic retinopathy retinal image using fuzzy C-means and morphological methods", proceedings of the third conference on international Conference: Advances in Computer science and Technology, Phuket, Thailand, pp. 359-364, April 02-04, 2007.

[3] Akara Sopharak, "Comparative Analysis of Automatic Exudates Detection between Machine Learning and Traditional Approaches", IEJCE Transaction of INF \& SYST, VOL.E92-D.NO.11. 2009, pp 2264-2271.

[4] H.F. Jaafar, A.K. Nandi and W. Al-Nauimy, "Automated detection of exudates in retinal images using a split-andmerge algorithm," EUSIPCO 2010, Alborg, pp. 16221626,2010

[5] Maria Garcia, Clara I. Sanchez, Jesus Poza, Maria I. Lopez and Roberto Hornero," Detection of Hard exudates In Retinal Images using A Radial Basis classifier", Annals Of Biomedical Engineering, Vol.37. No. 7. 2009. Pp 14481463

[6] R. Vijayamadheswaran, Dr. M. Arthanari, M.Sivakumar, "Detection Of Diabetic Retinopathy Using Radial Basis Function", International Journal Of Innovative Technology \& Creative Engineering, Vol.1 No.1.2011, pp 40-47.

[7] Neera Singh, Ramesh Chadra Tripathy, "Automates Early Detection Using Image Analysis Techniques", International Journal Of Computer Applications Volume 8-No.2, 2010, pp 18-23

[8] Nathan Silberman, Krity Ahlrich, Rob Fergus and Lakshminarayanan Subramanian, "Case for Automated Detection of Diabetic Retinopathy", Association for Advancement of Artificial Intelligence, 2010. 
[9] Vijayakumari,N.SuriyaNaraynan,"Diabetic RetinopathyEarly Detection Using Image Processing Techniques", International Journal On Computer Science and Engineering, Vol 2, No. 02, 2010, pp 357-361

[10] Asha Gowda karegouda, Asfiya Nasiha , M.A. Jayaram, "Exudates Detection in Retinal Images using Back propagation Neural Networks", International Journal Of Computer Applications, Volume 25-No 3, pp 25-31, 2011.

[11] Ivan Soares, Miguel Castelo-Branco, Antonio M, G Pinnheiro ," Exudates Dynamic Detection In Retinal Fundus Images Based On The Noise Map Distribution", 19th European Signal processing Conference 2011, pp 4650 .
[12] Celebi,M.E "Mining biomedical images with densitybased clustering" International conference on Information Technology; Coding and Computing, 2005, ITCC ,Vol 1 ,pp 163-168 .

[13] T.Kauppi, et.al "DIARETDB0: Evaluation database and morphology for diabetic retinopathy algorithm. Technical report, Lappeenranta University of Technology, Finland, 2006.

[14] T.Kauppi, et.al "DIARETDB1: diabetic retinopathy database and evaluation protocol," Technical report, Lappeenranta University of Technology of Kuopio, Finland, 2007. 